\title{
Vibration Analysis of Elastic Beams With Unconstrained Partial Viscoelastic Layer
}

\author{
Amirali Sadeqi and Shapour Moradi \\ Department of Mechanical Engineering, Shahid Chamran University of Ahvaz, Ahvaz, Iran.
}

\section{(Received 21 August 2015; accepted 5 October 2016)}

Material damping treatments, such as adding viscoelastic layers (VEL) to engineered structures, are commonly used for vibration attenuation. Study of the VEL geometry, shape, location (in case of partially adding), and arrangement (in composites), are of engineering interest to optimize the damping effect versus the weight and cost. In order to show the possibility of higher damping characteristics for shorter VEL, this paper aims for a two-step vibration analysis of an elastic cantilever beam with an unconstrained partial VEL. The governing equations are developed based on Euler-Bernoulli beam theory and Kelvin-Voigt viscoelastic model. In order to answer how the VEL length and thickness affect the modal parameters and dynamic response, both free and forced vibration problems are solved analytically, and the results are manipulated to achieve a much more applicable size range with higher damping characteristic. A non-uniform trend and inconsistent behaviour in both frequency and amplitude changes is observed versus increasing the VEL length, which addresses the necessity of an optimization challenge and gives a good insight to take into account the concept of VEL critical length.

\section{NOMENCLATURE}

$E_{e}, E_{v} \quad$ Elastic and viscoelastic Young's Modulus.

$H \quad$ Frequency response Function (FRF).

$I_{e}, I_{v} \quad$ Elastic and viscoelastic moment of inertia.

$k_{e}, k_{e v} \quad$ Elastic and composite (elastic-viscoelastic) bending stiffness.

$K(x) \quad$ Complex bending stiffness function.

$K_{e}, K_{e v} \quad$ Elastic and composite (elastic-viscoelastic) complex bending stiffness.

$l_{e} \quad$ Length of the elastic beam.

$m_{e}, m_{v} \quad$ Elastic and viscoelastic mass.

$p \quad$ Distributed force.

$p_{0} \quad$ Magnitude of harmonic excitation.

$q \quad$ Time dependent variable.

$r \quad$ Curvature radius.

$W \quad$ Spatial or time independent variable.

$w \quad$ Transverse displacement in $z$ direction.

$x_{v} \quad$ Length of the viscoelastic layer.

$\Gamma \quad$ Modal participation factor.

$\Omega \quad$ Excitation frequency.

$\varepsilon \quad$ Strain.

$\phi \quad$ Phase angle.

$\eta \quad$ Viscoelastic loss factor.

$\theta \quad$ Argument of the complex bending stiffness.

$\lambda_{1}, \lambda_{2} \quad$ Roots of characteristic equation.

$\rho \quad$ Mass density.

$\sigma \quad$ Stress.

$\omega \quad$ Natural frequency.

$\omega_{0}(x) \quad$ Stiffness to mass function.

$\omega_{e}, \omega_{e v} \quad$ Elastic and composite(elastic-viscoelastic)

stiffness to mass ratio.

$\psi \quad$ Quotient of the excitation frequency

to the function of stiffness to mass.

Damping ratio.

\section{ACRONYMS}

$\begin{array}{ll}\text { ACLD } & \text { Active constrained layer damping. } \\ \text { FEM } & \text { Finite element method. } \\ \text { FRF } & \text { Frequency response function. } \\ \text { PCLD } & \text { Passive constrained layer damping. } \\ \text { VDM } & \text { Viscoelastic damping material. } \\ \text { VEL } & \text { Viscoelastic layer. }\end{array}$

\section{INTRODUCTION}

Material damping is one of the most inexpensive, achievable, safe, and efficient ways to mitigate the vibration of structures, especially in aircrafts, rotating machines, vehicles, and so on. Seeking the modal parameters, i.e., natural frequency, mode shape, and damping ratio, is reckoned as a main analysing object and priority issue to assess the structural properties. On the other hand, a study on the dynamic response for various types of excitation such as transient, harmonic, and random plays a significant role to deal with real case of loadings, so that can be deemed as an essential step and mandatory procedure of structural design. In recent years, utilizing VEL (e.g., glass type composites, polyurethane foams, polymers, rubbers, etc.) on account of dissipating abilities are widespread to access the vibration attenuation and absorbing the impact loads. At first glance, the principal mission aims to reduce acoustic noise in walls, dissipation in massive structures, such as towers accompanied by some friction and hysteresis effects, or foundation of rotary machines. ${ }^{1}$ Viscoelastic (VE) materials, similar to the discrete viscous dampers, have an inherent dissipation factor which can implicitly change by time, temperature, and elastic properties., ${ }^{2,3}$ Based on the VE properties mentioned above, some of the laminates are basically composed of elastic and VELs to improve the strength and damping properties simultaneously. Therefore, one may take into account the optimization of the geometry and location of the VEL added to the elastic host structures. Moreover, plenty of structures which can be considered as beam structures with a 
clamped edge, (e.g., aircraft wing, tower crane jib, power line pile) may be exposed to long-term oscillation, while there is no possibility of attaching the VEL over the entire span of the beam. Hence, this justifies the idea of utilizing partial VEL in this type of structure.

In this article, of those conditions mentioned above, the effects of the length and thickness of attached VEL on free and forced vibrations of cantilever beam is investigated, to evaluate whether adding a larger unconstrained VEL improves the beam's damping characteristics or not. The word "unconstrained" accentuates taking advantage of bending effects rather than shear deformation of the VEL as a core between two elastic layers (constrains) and is very common in the literature.

During the past few decades, a lot of research has been carried out on dynamic analysis of structures with added VEL, and several numerical and experimental approaches have been developed. Zhou et al. ${ }^{38}$ reviewed various research methods and the theory calculation models that are employed in engineering to study the static and dynamic vibration characteristics of viscoelastic damping materials (VDM). Rearranging and optimizing the VELs within sandwich panels or composites is one of the interesting subjects which has been marked by some researchers. ${ }^{3,4,10-14}$ Some researchers have been focused on achieving higher energy dissipation, tuning, and vibration control for various compressional, bending, and sheer $^{3-7}$ loading cases, by means of analytical, numerical, ${ }^{8-18}$ and experimental ${ }^{10,19-22}$ methods. Vibration of structures considering VE damping effects under stochastic excitation have been surveyed in literature. ${ }^{23-25}$ Some authors have regarded either material or geometrical nonlinearities in the presence of VELs or VE dampers. ${ }^{12,25,26}$

Of the early studies on vibration of VE composites, is Kerwin's ${ }^{3}$ investigation for an elastic laminate with VE core using both theoretical and experimental method. Douglas and Yang ${ }^{4}$ investigated the effect of compressional damping in vibration of sandwich cantilevers composed of two elastic constraints bounding a VE core. Ardafio ${ }^{5}$ suggested a model containing a set of distributed VE hung cantilevers under a mass-spring system. Yamaguchi ${ }^{6}$ studied vibration of elastic beams with two built in edges, connected to a hung VE beam from mid span by a spring-damper. Inman $^{7}$ employed the method of separation of variables and used the kernel relaxation function to analyse a single VE beam. The laplace transformation process, based on orthogonality assumptions, was introduced afterwards to express the coupling between equations due to damping.

Adhikari ${ }^{8}$ proposed an approach for the model-order reduction of linear multiple degree of freedom VE systems via equivalent second order systems. Karim and Chen ${ }^{9}$ investigated the effects of surface damping between VELs and a simply supported elastic beam. Considering nonlinearities due to the tension, the transverse vibration of VE sandwich beam was studied by Haiwei et al. ${ }^{12}$ Lázaro et al. ${ }^{14}$ surveyed vibration of VE structures with proportional damping assumptions and extracted complex eigenvalues by utilizing the fixed point iteration method. Lázaro and Pérez ${ }^{15}$ studied the dynamics of frame structures with VEL and utilized fractional derivative to express constitutive relations. Eigenvalues were extracted and frequency response plotted for the cases with, and without, VEL. Eigenvalues of an Euler-Bernoulli beam supported by VE solids was studied by Cha et al. ${ }^{16}$ and their sensitivities were approximated by using Taylor series expansion. $\mathrm{Hu}$ and Wang ${ }^{17}$ investigated dynamics of viscoelastically damped structures by eliminating higher modes in frequency response, considered contribution of the lower modes, and the first two terms of the Neumann expansion of the unavailable modes. An iterative method, by means of normal modes, was used ${ }^{18}$ to calculate harmonic response of VE structures. Seismic behaviour and vibrational tests of steel frame structures with added VE dampers were experimentally investigated by Chang, ${ }^{19}$ Won Min, ${ }^{20}$ Bilbao, ${ }^{21}$ and their coworkers. Avcar ${ }^{30}$ and Saploğlu ${ }^{31}$ studied free vibration of steel beams with different boundary conditions and various geometrical characteristics, using Newton-Raphson and artificial neural network techniques. Effects of shear rotary inertia and nonhomogenous Young's modulus on natural frequencies of simply supported beam was also investigated by Avcar. ${ }^{32}$ An analytical model of the transient time response of an impacted cantilever beam with partial constrained layer damping (PCLD) was developed by Granger and Ross, ${ }^{33}$ and the effects of PCLD parameters on the initial transient response was studied. Following the recent work and case study, Blais et al. ${ }^{34}$ studied the suppression of non-causal effects, due to time aliasing, occurring when continuous frequency spectra are discretized.

Kumar and Singh ${ }^{35}$ examined the effect of parametric variation of active constrained layer damping (ACLD) on the vibration control of the beams. Optimum active or passive constrained layer damping (ACLD/PCLD) patches was placed by means of closed and open loop FEM model. El Hachemi et al., ${ }^{36}$ proposed an intuitive computational multi-scale homogenization procedure and tool for the estimation of the effective static and mechanical properties of complex viscoelastic composite material and structures. The proposed solution consisted of numerically computing the complex effective properties (storage and loss moduli) as a function of frequency. Høgsberg ${ }^{37}$ investigated the free vibration characteristics of structures with viscoelastic treatment by the complex-valued natural frequencies, and modified the assumed single mode by a correction term representing the influence of residual vibration modes.

The present work, investigates the effect of the VEL geometric parameters on the damping characteristic of a cantilever beam in free and forced vibration. Modal parameters of the structure, as well as harmonic resonant response, are studied to explain their behaviour over the changes of VEL parameters. The geometry of the problem is divided into two segments of composite elastic-VEL. The governing differential equations of motion are determined based on Euler-Bernoulli beam theory for composite elastic-VE segment, and then by applying a singular step function, it is generalized for all the domain, both the composite and elastic parts.

In Section 2.1, a separation of variable technique is used to solve the Eigen problem and to extract the modal parameters. To verify and compare the analytical natural frequencies and modes, and also to find an acceptable size of the structure in which Euler beam assumptions (analytical solution) are valid, an FEM simulation (3D) is also carried out by means of commercial software.

In Section 2.2, a harmonic analysis is exploited by assuming a harmonic excitation at the tip of the structure to obtain the response and comparing for various size of VEL length and thickness. 


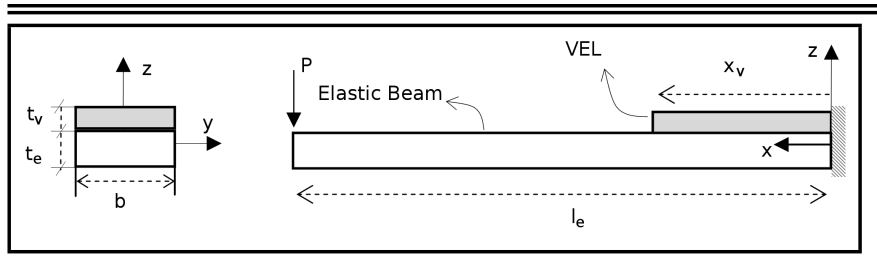

Figure 1. Schematic of the host structure, partial VEL and cross sections.

In Section 3, results are presented and discussed as tables and diagrams of natural frequencies, FRFs, phase angle, and effect of variation of VEL dimensions on damping characteristics. Finally, the summary is expressed and the results are concluded in Section 4.

\section{GOVERNING EQUATIONS}

As shown in Fig. (1), the geometry of the problem in which a partial unconstrained VEL is attached to an elastic cantilever beam (as the host or base structure), and loaded by a concentrated force at the free edge.

The elastic beam and the VEL attachment are assumed to be thin, so that the Euler-Bernoulli beam theory can be used. It is assumed that the elastic beam and VEL are completely tightened together therefore, there is no contact between them and shear forces, and delamination effects are neglected. No structural damping is considered except VEL loss effects. Considering the Kelvin-Voigt model for VEL, ${ }^{27}$ the stress-strain relationship can be expressed by the following relation:

$$
\sigma=E \varepsilon+\eta E d \varepsilon / d t
$$

where $\sigma$ and $\varepsilon$ are the stress and strain, $E$ is the Young modulus, and $\eta$ is the damping coefficient. In the case of bending of the VE beam, Hooke's law states

$$
\sigma_{x}=E_{v}^{*} \varepsilon_{x}
$$

where $E_{v}^{*}$ denotes the complex modulus of VE material as:

$$
E_{v}^{*}=E_{v}\left(1+\eta \frac{\partial}{\partial t}\right)
$$

Using the force and moment equilibrium equations, and straincurvature relation, one can express the general equation of motion along the structure by:

$$
\begin{array}{r}
\left(E_{e} I_{e}+E_{v} I_{v}\left\langle x_{v}-x\right\rangle^{0}\right) \frac{d^{4} w}{d x^{4}}+ \\
\left(\eta E_{v} I_{v}\left\langle x_{v}-x\right\rangle^{0}\right) \frac{d^{4} w}{d t d x^{3}}+ \\
\left(m_{e}+m_{v}\left\langle x_{v}-x\right\rangle^{0}\right) \frac{d^{2} w}{d t^{2}}=p(x, t) ;
\end{array}
$$

where $w$ represents the transverse deflection and subscript indices " $v$ " and " $e$ " denote VEL and elastic properties, respectively. $M$ and $I$ are mass per unit length and moment of inertia, and $p$ is the distributed load. The operator $\left\langle x_{v}-x\right\rangle^{0}$ is a step function, the value of which switches between 0 for $x_{v}<x \leq l_{e}$ and 1 for $0<x \leq x_{v}$, whereby the distribution of VE bending stiffness over the longitudinal direction can be piece-wisely figured.

\subsection{Free Vibration}

Solution of Eq. (4) in the absence of external loads results in an eigenvalue problem and the extraction of natural frequencies and mode shapes. Applying the method of separation of variables (i.e., $w(x, t)=W(x) \cdot T(t)$ ) to Eq. (4), one can obtain the following two differential equations:

$$
\begin{gathered}
\frac{d^{2} q}{d t^{2}}+2 \zeta \omega \frac{d q}{d t}+\omega^{2} q=0 \\
\frac{d^{4} W}{d x^{4}}-\left(\frac{\omega}{\omega_{0}(x)}\right)^{2} W=0 ;
\end{gathered}
$$

where $\omega$ is the natural frequency, and $\zeta$ and $\omega_{0}$ are the damping ratio and stiffness to the mass (inertia) ratio, respectively. Dividing the domain into elastic and composite (elastic-VEL) parts, the solution of Eq. (8), can be written as:

$$
\begin{array}{r}
W_{\mid 0<x \leq x_{v}}=W_{e v}=C_{11} \cos \lambda_{1} x+C_{12} \sin \lambda_{1} x \\
+C_{13} \cosh \lambda_{1} x+C_{14} \sinh \lambda_{1} x ; \\
W_{\mid x_{v}<x \leq l_{e}=} \begin{array}{r}
W_{e}=C_{21} \cos \lambda_{2} x+C_{22} \sin \lambda_{2} x \\
+C_{23} \cosh \lambda_{2} x+C_{24} \sinh \lambda_{2} x ;
\end{array}
\end{array}
$$

where subscript ' $e v$ ' and ' $e$ ' stand for elastic-VE and elastic parts, respectively, $\lambda_{1}$ and $\lambda_{2}$ are the roots of the characteristic equation of Eq. (6) which correspond to $\omega_{0}$ of each segment. $C_{i j}$ are the coefficients that can be determined using boundary condition. In order to find these coefficients, one should apply the four boundary conditions of the cantilever beam as:

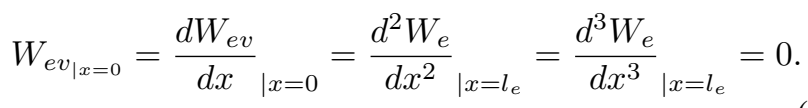

Besides, four continuity conditions are necessary to apply in transition region of jointing elastic to composite segment as $\left(x=x_{v}\right)$ :

$$
\begin{aligned}
W_{e v} & =W_{e} ; \\
\frac{d W_{e v}}{d x} & =\frac{d W_{e}}{d x} ; \\
k_{e v} \frac{d^{2} W_{e v}}{d x^{2}} & =k_{e} \frac{d^{2} W_{e}}{d x^{2}} ; \\
k_{e v} \frac{d^{3} W_{e v}}{d x^{3}} & =k_{e} \frac{d^{3} W_{e}}{d x^{3}} ;
\end{aligned}
$$

where $k_{e}$ and $k_{e v}$ denote the elastic and composite bending stiffness, respectively. Expressing Eq. (6) in terms of Eq. (7), and applying the conditions in Eqs. (8) and (9), leads to an algebraic set of equations shown by Eq. (10).

The determinant of the left-hand side matrix in the recent relation, must be zero for the existence of the non-trivial solution, which leads to a characteristic equation in terms of $\omega$. In fact, all the components of matrix are known by the natural frequency $\omega$. (i.e., each component is a trigonometric and hyperbolic functions of $\omega$ ). Hence, it is sufficient to enter the so-called known inputs and use an arbitrary determinant technique to obtain characteristic equation in terms of $\omega$, and then finding zeros or intersections, with the $x$ direction vector. In the present work, simple programming has been carried out in Maple-13 to obtain characteristic equation and zeros. 


$$
\begin{aligned}
& {\left[\begin{array}{cccc}
1 & 0 & 1 & 0 \\
0 & 1 & 0 & 1 \\
\cos \lambda_{1} x_{v} & \sin \lambda_{1} x_{v} & \cosh \lambda_{1} x_{v} & \sinh \lambda_{1} x_{v} \\
-\lambda_{1} \sin \lambda_{1} x_{v} & \lambda_{1} \cos \lambda_{1} x_{v} & \lambda_{1} \sinh \lambda_{1} x_{v} & \lambda_{1} \cosh \lambda_{1} x_{v} \\
-k_{e v} \lambda_{1}^{2} \cos \lambda_{1} x_{v} & -k_{e v} \lambda_{1}^{2} \sin \lambda_{1} x_{v} & k_{e v} \lambda_{1}^{2} \cosh \lambda_{1} x_{v} & k_{e v} \lambda_{1}^{2} \sinh \lambda_{1} x_{v} \\
k_{e v} \lambda_{1}^{3} \sin \lambda_{1} x_{v} & -k_{e v} \lambda_{1}^{3} \cos \lambda_{1} x_{v} & k_{e v} \lambda_{1}^{3} \sinh \lambda_{1} x_{v} & k_{e v} \lambda_{1}^{3} \cosh \lambda_{1} x_{v}
\end{array} \ldots\right.} \\
& \begin{array}{rrrr}
0 & 0 & 0 & 0
\end{array} \\
& \begin{array}{llll}
0 & 0 & 0 & 0
\end{array} \\
& 0 \quad 0 \quad 0 \\
& \left.\begin{array}{cccc}
0 & 0 & 0 & 0 \\
-\cos \lambda_{2} x_{v} & -\sin \lambda_{2} x_{v} & -\cosh \lambda_{2} x_{v} & -\sinh \lambda_{2} x_{v} \\
\lambda_{2} \sin \lambda_{2} x_{v} & -\lambda_{2} \cos \lambda_{2} x_{v} & -\lambda_{2} \sinh \lambda_{2} x_{v} & -\lambda_{2} \cosh \lambda_{2} x_{v} \\
k_{e} \lambda_{2}^{2} \cos \lambda_{2} x_{v} & k_{e} \lambda_{2}^{2} \sin \lambda_{2} x_{v} & -k_{e} \lambda_{2}^{2} \cosh \lambda_{2} x_{v} & -k_{e} \lambda_{2}^{2} \sinh \lambda_{2} x_{v} \\
-k_{e} \lambda_{2}^{3} \lambda_{2} \sin \lambda_{2} x_{v} & k_{e} \lambda_{2}^{3} \cos \lambda_{2} x_{v} & -k_{e} \lambda_{2}^{3} \sinh \lambda_{2} x_{v} & -k_{e} \lambda_{2}^{3} \cosh \lambda_{2} x_{v} \\
-k_{e} \cos \lambda_{2} l_{e} & -k_{e} \sin \lambda_{2} l_{e} & k_{e} \cosh \lambda_{2} l_{e} & k_{e} \sinh \lambda_{2} l_{e} \\
k_{e} \sin \lambda_{2} l_{e} & -k_{e} \cos \lambda_{2} l_{e} & k_{e} \sinh \lambda_{2} l_{e} & k_{e} \cosh \lambda_{2} l_{e}
\end{array}\right]\left[\begin{array}{l}
C_{11} \\
C_{12} \\
C_{13} \\
C_{14} \\
C_{21} \\
C_{22} \\
C_{23} \\
C_{24}
\end{array}\right]=0 ;
\end{aligned}
$$

\subsection{Forced Vibration}

Considering harmonic force $p_{0} e^{j \Omega t}$ at the tip of cantilever and dividing response to two segments (e.g., composite and only elastic region), one can take the response as the following form: ${ }^{39}$

$$
\begin{array}{r}
w(x, t)=W(x, \Omega) e^{j(\Omega t-\phi)}=\left(W_{e v}\left(0<x<x_{v}, \Omega\right)\right. \\
\left.+W_{e}\left(x_{v}<x<l_{e}, \Omega\right)\right) e^{j(\Omega t-\phi)}
\end{array}
$$

where $\Omega$ denotes excitation frequency, $\phi$ is the phase difference due to damping (ineffective in computations), $W$ is the response amplitude in terms of frequency and position, and $j$ denotes imaginary part. Applying the above solution to Eq. (4), results in the following equation:

$$
\begin{array}{r}
-\left(m_{e}+m_{v}\left\langle x_{v}-x\right\rangle^{0}\right) \Omega^{2} W+\left(E_{e} I_{e}+E_{v} I_{v}\left\langle x_{v}-x\right\rangle\right. \\
\left.+j \Omega \eta E_{v} I_{v}\left\langle x_{v}-x\right\rangle^{0}\right) W_{, x x x x}=0 .
\end{array}
$$

It must be noted that the external force acts as the boundary load. Solution of the recent equation can be obtained by defining complex roots $\lambda$ as:

$$
\lambda_{n}=\sqrt[4]{\psi^{4} e^{j \theta}}=\psi e^{j\left(\frac{2 n \pi+\theta}{4}\right)}, \quad \mathrm{n}=0,1,2,3
$$

where $\psi$ can be defined by

$$
\psi^{4}(x)=\Omega^{2} / \frac{|K(x)|}{m(x)}
$$

where $K$ is complex bending stiffness with argument $\theta$ (see Ref. ${ }^{39}$ ). Assuming complex roots of Eq. (12), and no structural damping in the elastic segment, leads $\theta$ to be zero in this region and the solution can be expressed by the following complex form:

$$
\begin{aligned}
W_{e v}= & a_{1} \cos \left(\psi_{e v} \sin \left(\frac{\theta}{4}\right) x\right) \cosh \left(\psi_{e v} \cos \left(\frac{\theta}{4}\right) x\right) \\
& +a_{2} \cos \left(\psi_{e v} \cos \left(\frac{\theta}{4}\right) x\right) \sinh \left(\psi_{e v} \sin \left(\frac{\theta}{4}\right) x\right) \\
& +a_{3} \sin \left(\psi_{e v} \sin \left(\frac{\theta}{4}\right) x\right) \sinh \left(\psi_{e v} \cos \left(\frac{\theta}{4}\right) x\right) \\
& +a_{4} \sin \left(\psi_{e v} \cos \left(\frac{\theta}{4}\right) x\right) \cosh \left(\psi_{e v} \sin \left(\frac{\theta}{4}\right) x\right)
\end{aligned}
$$

$W_{e}=a_{5} \cos \psi_{e} x+a_{6} \sin \psi_{e} x+a_{7} \cosh \psi_{e} x+a_{8} \sinh \psi_{e} x$

where $\psi_{e}$ and $\psi_{e v}$ correspond to the elastic and composite segments in Eq. (14), respectively. In a similar manner to the previous section, after applying the boundary and continuity conditions, constants $a_{1}$ to $a_{8}$ can be obtained from the following algebraic set of equations, presented in Eq. (16), where $p_{0}$ is the magnitude of concentrated harmonic load at the tip of elastic beam, $K_{e v}$ and $K_{e}$ are complex and real bending stiffness corresponding to the composite and elastic segments, respectively. Rest of the symbols are defined by:

$$
\begin{gathered}
c=\cos \\
s=\sin \\
c h=\cosh \\
s h=\sinh \\
\bar{c}=\psi_{e v} \cos \left(\frac{\theta}{4}\right) \\
\bar{s}=\psi_{e v} \sin \left(\frac{\theta}{4}\right) .
\end{gathered}
$$

Solving the above complex system results in the frequency response function (FRF) and phase of the structure. 


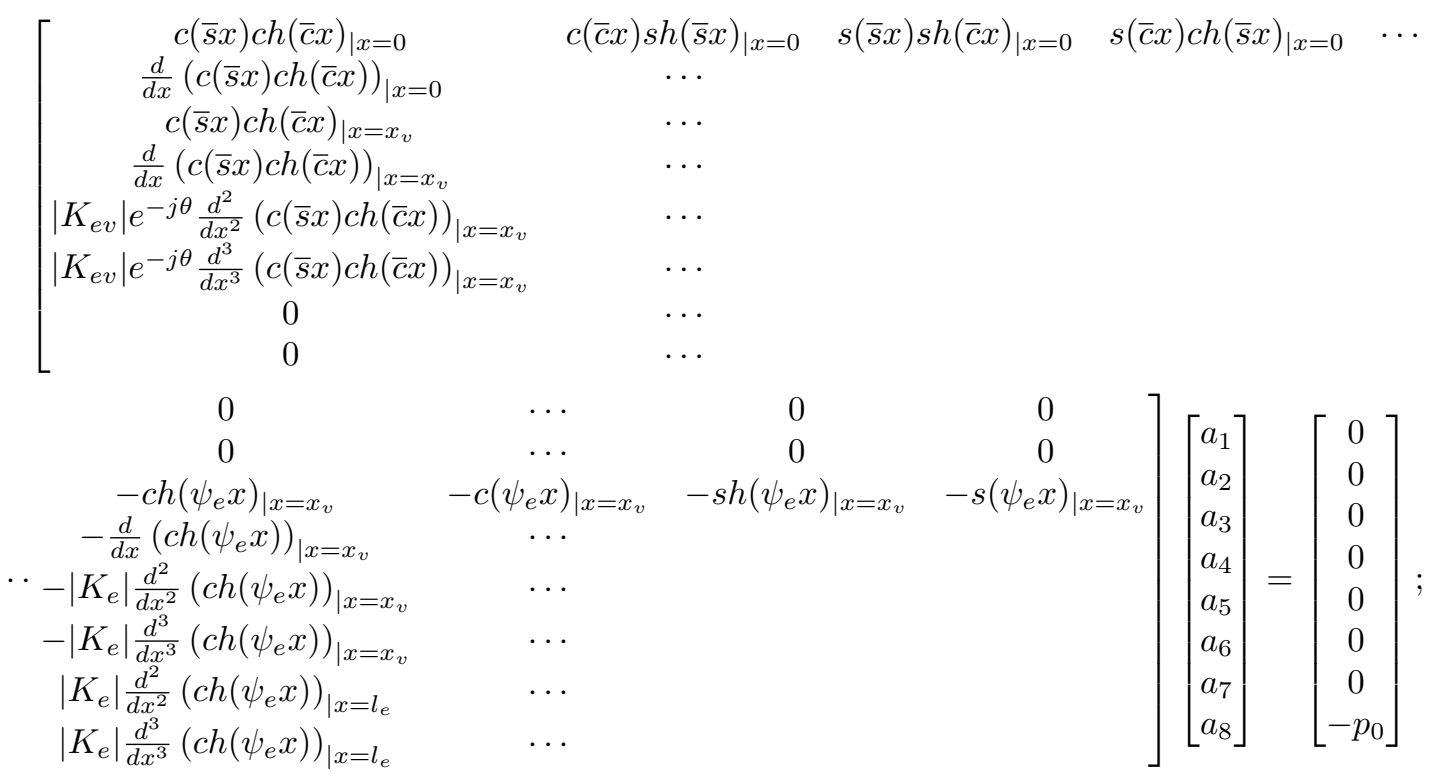

Table 1. Natural frequencies for different VEL length and thickness $\left(t_{e}=0.04 \mathrm{~m}, l_{e}=2 \mathrm{~m}\right)$.

\begin{tabular}{|c|c|c|c|c|c|c|c|c|c|c|}
\hline \multicolumn{3}{|c|}{ Error \% } & \multicolumn{3}{|c|}{ FEM } & \multicolumn{3}{|c|}{ Analytical } & \multicolumn{2}{|c|}{ Frequency $(\mathrm{Hz})$} \\
\hline $3^{\text {rd }}$ & $2^{\text {nd }}$ & $1^{\text {st }}$ & $3^{\text {rd }}$ & $2^{\text {nd }}$ & $1^{\text {st }}$ & $3^{\text {rd }}$ & $2^{\text {nd }}$ & $1^{\text {st }}$ & & Dimensions (m) \\
\hline 0.13 & 0.03 & 0.04 & 153.55 & 56.24 & 9.23 & 153.75 & 56.22 & 9.23 & $t_{v}=0.01$ & \\
\hline 0.43 & 0.38 & 0.56 & 161.67 & 59.42 & 9.74 & 162.37 & 59.65 & 9.79 & $t_{v}=0.02$ & $x_{v}=0.25$ \\
\hline 1.24 & 1.18 & 1.30 & 172.25 & 62.77 & 10.18 & 174.39 & 63.51 & 10.31 & $t_{v}=0.04$ & \\
\hline 1.84 & 1.66 & 1.61 & 177.34 & 64.18 & 10.35 & 180.60 & 65.25 & 10.52 & $t_{v}=0.06$ & \\
\hline 0.21 & 0.19 & 0.03 & 152.65 & 57.22 & 10.15 & 152.98 & 57.11 & 10.15 & $t_{v}=0.01$ & \\
\hline 0.37 & 0.03 & 0.43 & 160.11 & 62.94 & 11.46 & 160.70 & 62.92 & 11.51 & $t_{v}=0.02$ & $x_{v}=0.5$ \\
\hline 0.56 & 0.63 & 1.34 & 177.53 & 72.87 & 12.88 & 178.53 & 73.30 & 13.05 & $t_{v}=0.04$ & \\
\hline 1.04 & 1.4 & 1.84 & 196.81 & 79.30 & 13.51 & 198.85 & 80.41 & 13.76 & $t_{v}=0.06$ & \\
\hline 0.27 & 0.09 & 0.25 & 162.73 & 58.21 & 11.26 & 163.18 & 58.26 & 11.23 & $t_{v}=0.01$ & \\
\hline 0.71 & 0.46 & 0.07 & 179.73 & 62.51 & 14.21 & 181.00 & 62.80 & 14.22 & $t_{v}=0.02$ & $x_{v}=1$ \\
\hline 1.96 & 0.76 & 0.86 & 203.77 & 69.56 & 19.52 & 207.76 & 70.09 & 19.68 & $t_{v}=0.04$ & \\
\hline 3.01 & 0.73 & 1.72 & 215.35 & 77.61 & 23.55 & 221.83 & 78.18 & 23.96 & $t_{v}=0.06$ & \\
\hline 0.79 & 0.05 & 0.33 & 172.5 & 65.87 & 11.20 & 171.13 & 65.91 & 11.16 & $t_{v}=0.01$ & \\
\hline 1.08 & 0.59 & 0.13 & 192.98 & 79.07 & 14.22 & 195.06 & 79.53 & 14.20 & $t_{v}=0.02$ & $x_{v}=1.5$ \\
\hline 7.77 & 2.06 & 0.037 & 221.65 & 101.4 & 20.54 & 238.85 & 103.48 & 20.53 & $t_{v}=0.04$ & \\
\hline 3.15 & 3.74 & 0.14 & 247.91 & 116.83 & 27.06 & 255.73 & 121.2 & 27.10 & $t_{v}=0.06$ & \\
\hline
\end{tabular}

\section{RESULTS AND DISCUSSIONS}

To verify the analytical formulations presented in previous section, several numerical case studies were examined. The analytical solution was carried out using MAPLE software, and compared with the finite element results using commercial software. An aluminium cantilever beam having $40 \mathrm{~mm} \times 40 \mathrm{~mm}$ cross section and $2000 \mathrm{~mm}$ length, is considered. The beam has a Young 's modulus of $70 \mathrm{Gpa}$, Poisson's ratio of 0.33 and density of $2700 \mathrm{~kg} / \mathrm{m}^{3}$. The VEL material is made of G-11/Glass (see Ref. ${ }^{28,29}$ ) with modulus of elasticity, density, and poison's ratio of $181 \mathrm{Gpa}, 1500 \mathrm{~kg} / \mathrm{m}^{3}$ and 0.3 , respectively. It has the same cross sectional area as the aluminium beam with a variable length. The finite element model consisted of 100 solid elements for the beam and another 100 solid elements for the attached VEL. The analytical and FEM solutions has been carried out by Maple-13 and ANSYS-APDL12, respectively. The corresponding first three natural frequencies of the beam with attached VEL are presented in Table 1, for some particular characteristics and geometries. As seen from the table, the results obtained by the analytical method are in very good agreement with those obtained by FEM.

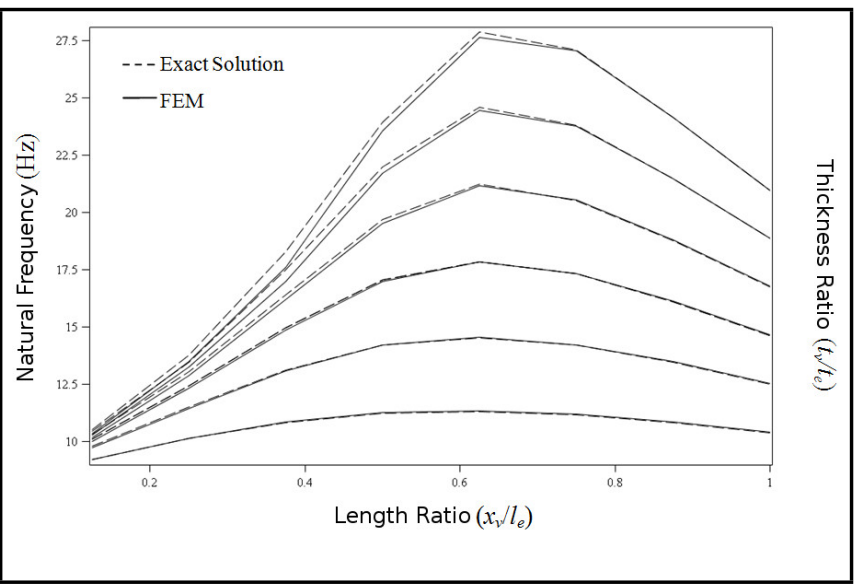

Figure 2. Variation of the first natural frequency versus the VEL size for $t_{e}=0.04 \mathrm{~m}, l_{e}=2 \mathrm{~m}$.

The analytical and FEM results are also presented in Fig. (2), with different length and thickness ratios. Since the structure has been modelled using 3-D solid elements, increasing the VEL thickness, causes some discrepancies compared to 1$\mathrm{D}$ analytical results. Based on finite strip (wide beam) and shear deformation (thick beam) theories, restrictions of appli- 
cable size for which the exact solution is valid can be appropriated for the length and thickness of VELs with errors less than 1 percent.

As demonstrated in Fig. (2), there is an acceptable alignment in two diagrams for relatively long and thin partial VEL. Comparisons of the first three mode shapes between FEM and the analytical solutions are shown in Fig. (3). Only the transverse modes of the 3D modelling in ANSYS are considered here. The results illustrated in Fig. (4) point out the frequency ratio diagram versus the VEL size variation. Hence, increasing the VEL length up to the half of the elastic beam span, causes an increase in fundamental frequency (dashed line) where beyond this region it starts to decrease. Since the fundamental frequency of a cantilever beam is proportional to the inverse of the square length, a frequency decline is expected due to increasing the length. But, in the discussed structure (elastic-VEL composite), fundamental frequency growing due to length increasing (for a length ratio less than 0.6) is related to the contribution of VEL and the amount of its participation to the response. On the other hand, the effect of increasing the total thickness of a structure (composite segment) is dominant in comparison with increasing the VEL length. This is more observable in higher thickness ratios, as shown in the diagram. The diagram for the second mode (dashed-point line) is clustered to one valley and two peaks on both sides, and maximum frequency occurs almost at the quarter and three quarters of length ratios.

In general, adding partial VEL to an elastic beam, causes an increase in the thickness and length of the composite segment; the former will result an increase in natural frequencies, while the latter will cause the reduction of natural frequencies. Therefore, it is either a thickness or length increase that dictates how the frequency must change, so that reconciliation makes maximum frequency for length ratio about 0.6 , and for any arbitrary geometrical size of the elastic part and VEL thickness, where shown in Fig. (4) and Fig. (5). According to the stiffness to mass ratios $\omega_{e v}, \omega_{e}$, corresponding to the elastic and composite segments respectively, one can consider this as a scale or evaluation criterion of natural frequencies in structures, so that multiplying each by participation factors:

$$
\begin{gathered}
\Gamma_{e v}=\left(\int_{0}^{x_{v}} \frac{W_{e v}^{I V}}{W_{e v}} d x\right)^{\frac{1}{2}} ; \\
\Gamma_{e}=\left(\int_{x_{v}}^{l_{e}} \frac{W_{e}^{I V}}{W_{e}} d x\right)^{\frac{1}{2}} ;
\end{gathered}
$$

results in exactly the natural frequencies (i.e., $\omega=$ $\left.\left(\Gamma_{e v} \omega_{e v}\right)_{\mid x=x_{v} / l_{e}}=\left(\Gamma_{e} \omega_{e}\right)_{\mid x=x_{v} / l_{e}}\right)$. Increasing the length ratio causes a decrease in $\omega_{e v}$ and an increase $\omega_{e}$, respectively, and that is the corresponding participation factor which specifies quantity of the natural frequency as a function of geometry. As a result, for the length ratio about 0.6 , this is the aforesaid factor which determines destiny of the maximum frequency in this region.

The magnitude of FRF at the tip of the structure is shown in Fig. (6), for various loss factors. In higher frequency range, utilizing VEL even with a poor loss factor, makes a significant decrease in the response amplitude. For the first resonant frequency of $14.22 \mathrm{~Hz}$ and a loss factor of $\eta=0.047$, damping ratio equals to the critical value of $\zeta=1$ and therefore, for frequencies or loss factors a bit more than these, over damping occurs.

Impedance phase angles concerning the first three modes are presented in Fig. (7). As seen from the figure, there is a $2 \pi$ difference between responses with higher and less than first resonant mode. The figure also shows that, the next $2 \pi$ phase differences do not occur exactly at the $2^{\text {nd }}$ and $3^{\text {rd }}$ resonant modes (e.g., $62.80 \mathrm{~Hz}$ and $181.00 \mathrm{~Hz}$ ), because, as mentioned, the critical damping appears for the cases with lower loss factors. However, varying the dimension of the VEL makes no instrumental sense to compare and evaluate the results to deduct which size of VEL represents higher damping performance. Hence, it will be helpful utilizing the FRF peak at resonant mode of each size (rather than expressing in terms of frequency) and then comparing to each other.

Figure (8), shows the variation of the ratio of the FRF peak at the first resonant mode $H\left(\omega_{1}\right)$, to static level $H(0)$, for various length ratios at several thickness ratios.

For any VEL size, minimum ratio happens when the length ratio is rising to about 0.6 ; i.e., for VEL with a length about half (or a little more than half) of the size of the elastic beam. The diagrams for various thicknesses in this region $\left(x_{v} / l_{e}<0.6\right)$ are descending and, in spite of intersecting each other at some particular length ratios, totally increasing the thickness has a positive effect in vibration amplitude reduction.

As a matter of fact, a thicker structure naturally has a lower transverse deflection, therefore, intersections can be depicted such that the static response or denominator of the ratio, is always descending versus increasing the thickness or length ratio. On the other hand, although the numerator (first resonant response) decreases as the length ratio increases, for the length ratio less than 0.6 , the static stiffness has a dominant effect on decaying the amplitude than the dynamic damping. Thus, the numerator does not decrease as much as the denominator in this region. Nevertheless, it does not mean the smaller thickness of VEL is better in decaying the resonant amplitude.

Contrarily, for a length ratio more than about half $\left(x_{v} / l_{e}>0.6\right)$, meaning longer VEL up to the elastic length, the amplitude begins to increase and there is no intersection for various thicknesses in this region. It simply means using a larger VEL does not necessarily guarantee a further amplitude decay, though, it contributes to decrease the total static deflection and the strength of structure.

\section{CONCLUSIONS}

The modal parameters of an elastic beam with a partial unconstrained VEL were analytically obtained based on the Kelvin-Voigt VEL model and the Euler-Bernoulli beam theory. Applicable size and dimension range for which analytical solutions and assumptions have been valid, were detected by comparing to the FEM results.

An irregular variation was observed in natural frequencies versus VEL size. It was shown that the natural frequencies are proportional to the stiffness to mass ratio, as well as the participation factor related to the contribution of the VEL length in mode shapes. 


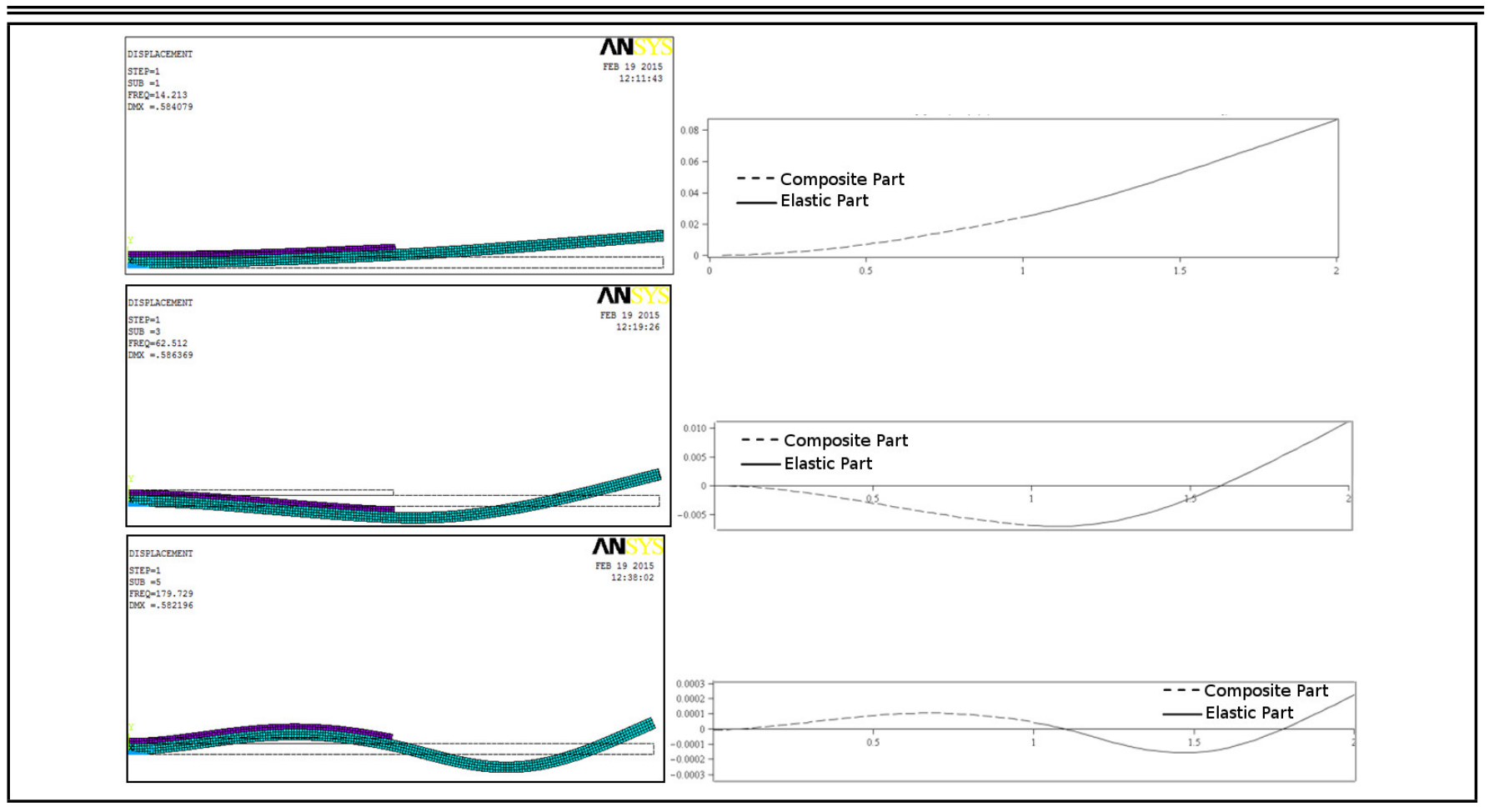

Figure 3. The first three transverse mode shapes, $t_{v}=0.02 \mathrm{~m}, x_{v}=1 \mathrm{~m}$, Left: FEM, Right: Analytical.

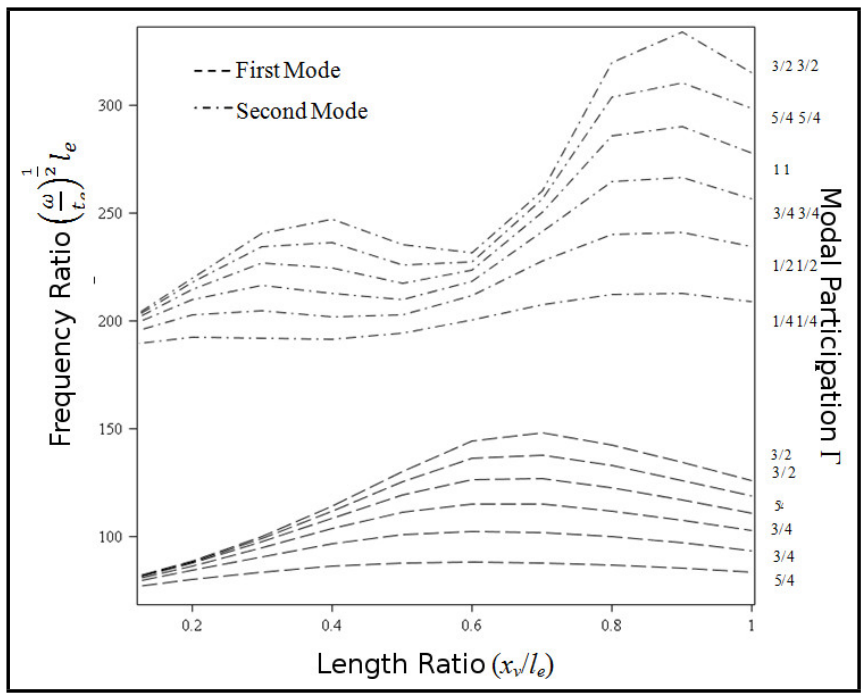

Figure 4. Variation of the frequency ratio versus the length for various thickness ratios.

The fundamental frequency increases via enlarging the VEL to about 60 percent of the elastic beam span, whereas by lengthening more than this, the frequency gradually begins to decrease. As a result, adding a larger VEL does not necessarily increase the fundamental frequency.

Moreover, frequency response, phase angle, and first resonant FRF ratio, were analytically extracted. It was demonstrated that the amplitude of the structure response always decreases by increasing the loss factor as well as VEL thickness. The first resonant peak meets its minimum for VEL length of about 0.6 of the elastic beam span, whereas for longer VEL, it shows a growing amplitude because of the higher participation of longer VEL in this region, where decreases the natural frequency and damping ratio.

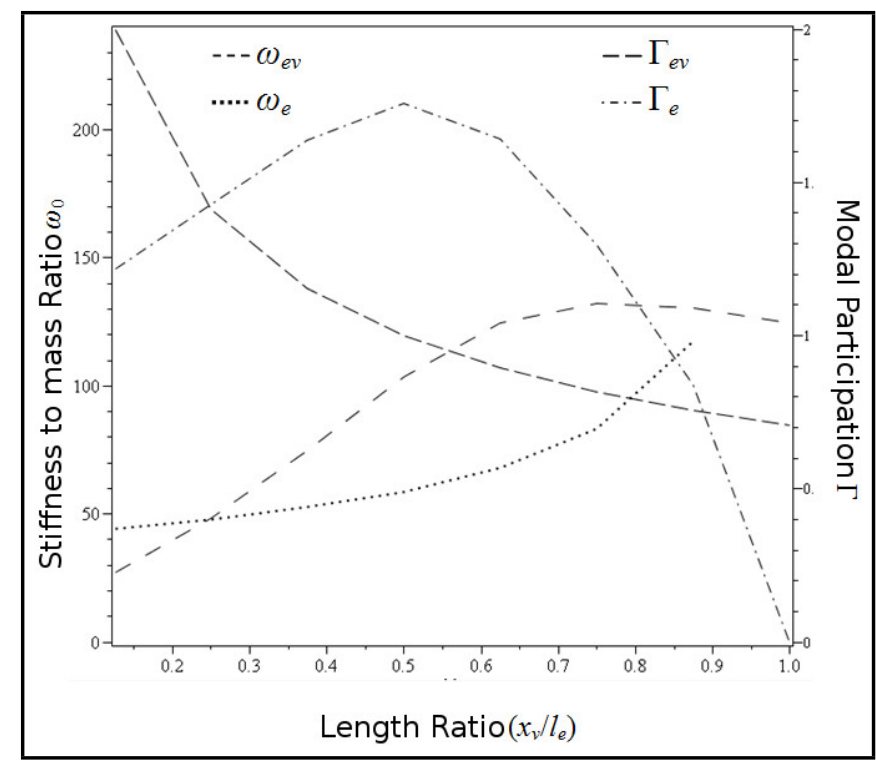

Figure 5. Variation of stiffness to mass ratio and participation factor versus the VEL size.

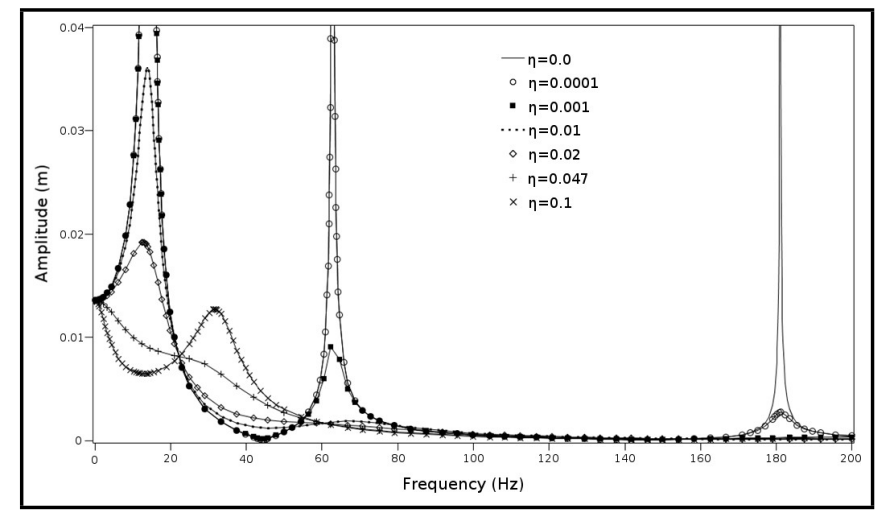

Figure 6. The response amplitude at the tip of the beam for various loss factors and $t_{v}=0.02 \mathrm{~m}, x_{v}=1 \mathrm{~m}$. 


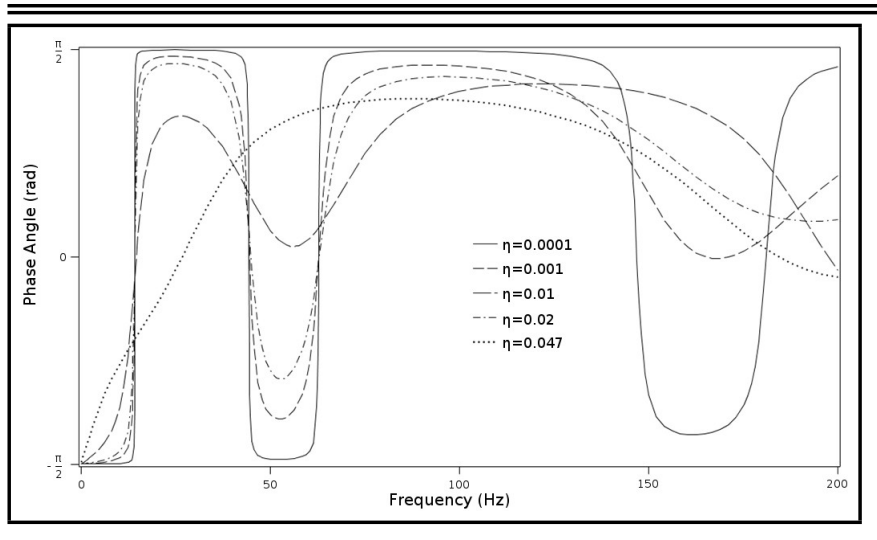

Figure 7. The response phase at the tip of the beam for various loss factors and $t_{v}=0.02 \mathrm{~m}, x_{v}=1 \mathrm{~m}$

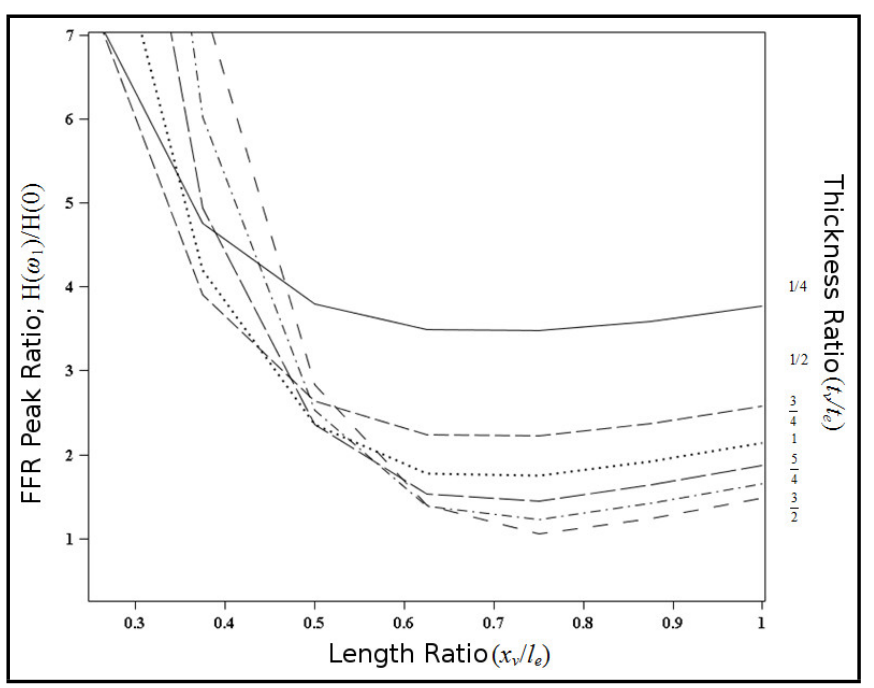

Figure 8. Variation of the ratio of FRF peak to static level $H(0)$ versus the VEL size.

\section{REFERENCES}

1 Chen, C. P. and Lakes, R. S. Design of viscoelastic impact absorbers: optimal material properties, Journal of Solids and Structures, 26 (12), 1313-1328, (1990). https://dx.doi.org/10.1016/0020-7683(90)90081-6

2 Sarhadi, A., Hattel, J. H., and Hansen, H. N. Evaluation of the viscoelastic behaviour and glass/mould interface friction coefficient in the wafer based precision glass moulding, Journal of Materials Processing Technology, 214, 1427-1435, (2014). https://dx.doi.org/10.1016/j.jmatprotec.2014.02.008

3 Kerwin, E. M. Damping of flexural waves by a constrained viscoelastic layer, Journal of the Acoustical Society of America, 31 (7), 952-962, (1959). https://dx.doi.org/10.1121/1.1907821

4 Douglas, B. E. and Yang, J. C. Transverse compressional damping in the vibratory response of elasticviscoelastic-elastic beams, American Institute of Aeronautics and Astronautics Journal, 16 (9), 925-930, (1978). https://dx.doi.org/10.2514/3.7595

5 Ardayfio, D. Viscoelastic modeling of cantilever vibration absorbers, Mechanical Modeling, 3, 183-189, (1982). https://dx.doi.org/10.1016/0270-0255(82)90023-9
6 Yamaguchi, H. Vibrations of a beam with an absorber consisting of a viscoelastic beam and a spring-viscous damper, Journal of Sound and Vibration, 103 (3), 417-425, (1985). https://dx.doi.org/10.1016/0022-460X(85)90432-8

7 Inman, D. J. Vibration analysis of viscoelastic beams by separation of variables and modal analysis, Mechanics Research Communications, 16 (4), 213-218, (1989). https://dx.doi.org/10.1016/0093-6413(89)90025-6

8 Adhikari, S. A reduced second-order approach for linear viscoelastic oscillators, Journal of Applied Mechanics, 77, 041003-1, (2010). https://dx.doi.org/10.1115/1.4000913

9 Karim, K. R. and Chen, G. D. Surface damping effect of anchored constrained viscoelastic layers on the flexural response of simply supported structures, Mechanical Systems and Signal Processing, 27, 419-432, (2012). https://dx.doi.org/10.1016/j.ymssp.2011.09.006

10 Wang, Y. and Inman, D. J. Finite element analysis and experimental study on dynamic properties of a composite beam with viscoelastic damping, Journal of Sound and Vibration, 332, 6177-6191, (2013). https://dx.doi.org/10.1016/j.jsv.2013.06.016

11 Moita, J. S., Araújo, A. L., MotaSoares, C. M., and MotaSoares, C. A. Finite element model for damping optimization of viscoelastic sandwich structures, $A d$ vances in Engineering Software, 66, 34-39, (2013). https://dx.doi.org/10.1016/j.advengsoft.2012.10.002

12 Haiwei, L., Yinghui, L., Liang, L., and Qikuan, L. Transverse vibration of viscoelastic sandwich beam with timedependent axial tension and axially varying moving velocity, Applied Mathematical Modelling, 38, 2558-2585, (2014). https://dx.doi.org/10.1016/j.apm.2013.10.055

13 Aumjaud, P., Smith, C. W., and Evans, K. E. A novel viscoelastic damping treatment for honeycomb sandwich structures, Composite Structures, 119, 322-332, (2015). https://dx.doi.org/10.1016/j.compstruct.2014.09.005

14 Lázaro, M., Pérez-Aparicio, J., and Epstein, M. Computation of eigenvalues in proportionally damped viscoelastic structures based on the fixed-point iteration, Applied Mathematics and Computation, 219 (8), 3511-3529, (2012). https://dx.doi.org/10.1016/j.amc.2012.09.026

15 Lázaro, M. and Pérez-Aparicio, J. Dynamic analysis of frame structures with free viscoelastic layers: new closed-form solutions of eigenvalues and a viscous approach, Engineering Structures, 54, 69-81, (2013). https://dx.doi.org/10.1016/j.engstruct.2013.03.052

16 Cha, P. D., Carbon, K. C., and Hsieh, R. Eigenvalues and eigenvalue sensitivities of a beam supported by viscoelastic solids, Journal of Vibration and Acoustics, 136 (2), $021017-$ 1, (2014). https://dx.doi.org/10.1115/1.4026175

17 Li, L., Hu, L.Y., and Wang, X. Eliminating the modal truncation problem encountered in frequency responses of viscoelastic systems, Journal of Sound and Vibration, 333 (4), 1182-1192, (2014). https://dx.doi.org/10.1016/j.jsv.2013.10.018 
$18 \mathrm{Li}, \quad$ L., Hu, L. Y., and Wang, X. Harmonic response calculation of viscoelastic structures using classical normal modes: an iterative method, Computers and Structures, 133, 39-50, (2014). https://dx.doi.org/10.1016/j.compstruc.2013.11.009

19 Chang, K. C., Lai, M. L., Soong, T. T., Hao, D. S., and Yeh, Y. C. Technical Report NCEER-93-0009, Seismic Behavior and Design Guidelines for Steel Frame Structures with Added Viscoelastic Dampers, (1993).

20 Won Min, K., Kimb, J., and Lee, S. Vibration tests of 5-storey steel frame with viscoelastic dampers, Engineering Structures, 26 (6), 831-839, (2004). https://dx.doi.org/10.1016/j.engstruct.2004.02.004

21 Bilbao, A., Avilés, R., Agirrebeitia, J., and Ajuria, G. Proportional damping approximation for structures with added viscoelastic dampers, Finite Elements in Analysis and Design, 42 (6), 492-502, (2006). https://dx.doi.org/10.1016/j.finel.2005.10.001

22 Ghiringhelli, G. L. and Terraneo, M. Analytically driven experimental characterisation of damping in viscoelastic materials, Aerospace Science and Technology, 40, 75-85, (2015). https://dx.doi.org/10.1016/j.ast.2014.10.011

23 Palmeri, A. Correlation coefficients for structures with viscoelastic dampers, Engineering Structures, 28 (8), 1197-1208, (2006). https://dx.doi.org/10.1016/j.engstruct.2005.12.015

24 Guedri, M., Lima, A. M., Bouhaddi, N., and Rade, D. A. Robust design of viscoelastic structures based on stochastic finite element models, Mechanical Systems and Signal Processing, 24 (1), 59-77, (2010). https://dx.doi.org/10.1016/j.ymssp.2009.03.010

$25 \mathrm{Xu}, \mathrm{Y}$., Li, Y., and Liu, D. Response of fractional oscillators with viscoelastic term under random excitation, Journal of Computational and Nonlinear Dynamics, 9 (3), 031015-1, (2014). https://dx.doi.org/10.1115/1.4026068

26 Mahmoodi, S. N., Khadem, S.E., and Kokabi, M. Non-linear free vibrations of kelvin-voigt viscoelastic beams, International Journal of Mechanical Sciences, 49 (6), 722-732, (2007). https://dx.doi.org/10.1016/j.ijmecsci.2006.10.005

27 Flugge, W. Viscoelasticity, 2nd Ed, Springer-Verlag, Berlin, Germany, (1975). https://dx.doi.org/10.1007/978-3662-02276-4

28 Scherer, G. and Rekhson, S. Viscoelastic-Elastic composites: II, sandwich seal, 83rd Annual Meeting, the American Ceramic Society, Glass Division Nos. 60G-XI and 61-G-81, Washington, DC, May 8, (1981). https://dx.doi.org/10.1111/j.1151-2916.1982.tb10492.x

29 Soules, T., Busbey, R., Rekhson, S., Markkovsky, A., and Burkey, M. Finite Element Calculations of Residual Stresses in Glass Parts Using MARC, General Electric Company (Nela Park), Report: 86-LRL-2022, Cleveland, OH, March (1986). https://dx.doi.org/10.1111/j.11512916.1987.tb04935.x
30 Avcar, M. Free vibration analysis of beams considering different geometric characteristics and boundary conditions, International Journal of Mechanics and Applications, 4 (3), 94-100, (2014). https://dx.doi.org/10.5923/j.mechanics.20140403.03

31 Avcar, M. and Saploğlu, K. An artificial neural network application for estimation of natural frequencies of beams, International Journal of Advanced Computer Science and Applications, 6 (6), 94-102, (2015). https://dx.doi.org/10.14569/IJACSA.2015.060614

32 Avcar, M. Effects of rotary inertia shear deformation and non-homogeneity on frequencies of beam, Structural Engineering and Mechanics, 55 (4), 871-884, (2015). https://dx.doi.org/10.12989/sem.2015.55.4.871

33 Granger, D. and Ross, A. Effects of partial constrained viscoelastic layer damping parameters on the initial transient response of impacted cantilever beams: Experimental and numerical results, Journal of Sound and Vibration, 321 (1-2), 45-64, (2009). https://dx.doi.org/10.1016/j.jsv.2008.09.039

34 Blais, J. F., Cimmino, M., Ross, A., and Granger, D. Suppression of time aliasing in the solution of the equations of motion of an impacted beam with partial constrained layer damping, Journal of Sound and Vibration, 326 (3-5), 870882, (2009). https://dx.doi.org/10.1016/j.jsv.2009.06.001

35 Kumar, N. and Singh, S. P. Vibration and damping characteristics of beams with active constrained layer treatments under parametric variations, $M a$ terials and Design, 30 (10), 4162-4174, (2009). https://dx.doi.org/10.1016/j.matdes.2009.04.044

36 El Hachemi, M., et al. An intuitive computational multi-scale methodology and tool for the dynamic modeling of viscoelastic composites and structures, Composite Structures, 144, 131-137, (2016). https://dx.doi.org/10.1016/j.compstruct.2016.02.032

37 Høgsberg, J. Explicit solution for the natural frequency of structures with partial viscoelastic treatment, Mechanics of Advanced Materials and Structures, (2015), In press. https://dx.doi.org/10.1080/15376494.2015.1029166

38 Zhou, X. Q., Yu, D. Y., Shao, X. Y., Zhang, S. Q., and Wang, S. Research and applications of viscoelastic vibration damping materials: A review, Composite Structures, (2015), In press. https://dx.doi.org/10.1016/j.compstruct.2015.10.014

39 Sadeqi, A. and Moradi, S. Geometrical effects of viscoelastic layer added to an elastic beam on forced vibration response, Proc. 5th International Conference on Acoustics \& Vibration, ISAV, University of Tehran, Iran, (2015). 